\title{
Plasma Fibrinogen Along with Patient-reported Outcome Measures Enhances Management of Polymyalgia Rheumatica: A Prospective Study
}

\author{
Eoghan M. McCarthy, Paul A. MacMullan, Shibeb Al-Mudhaffer, Anne Madigan, \\ Suzanne Donnelly, Conor J. McCarthy, Eamonn S. Molloy, Dermot Kenny, \\ and Geraldine M. McCarthy
}

ABSTRACT. Objective. We sought to prospectively examine the responsiveness of a number of patient-reported outcome (PRO) measures in polymyalgia rheumatica (PMR), as well as their relationship to the biomarkers erythrocyte sedimentation rate (ESR), C-reactive protein (CRP), and plasma fibrinogen. Methods. Sixty patients with PMR were divided into active $(n=25)$ or inactive $(n=35)$ disease groups based on symptoms; physician assessment; and the biomarkers ESR, CRP, and plasma fibrinogen. Groups underwent assessment at baseline and 6 weeks. Disease activity measures and relevant PRO measures were recorded. Measures of responsiveness were compared for all PRO and biomarkers.

Results. Visual analog scale disease activity (VASDA) and VAS quality of life (VASQOL) are more responsive to change in disease activity than VAS pain, morning stiffness, Health Assessment Questionnaire (HAQ), and PMR-activity score (AS). Analysis of PMR-AS versus VASDA, VASQOL, and HAQ showed correlation coefficients of $0.87(\mathrm{p}<0.001), 0.80(\mathrm{p}<0.001)$, and 0.68 ( $p<0.001)$, respectively. Receiver-operating curve (ROC) analysis revealed VASDA to be more specific than either HAQ ( 0.95 vs 0.85 ; $p<0.001)$ or VASQOL $(0.95$ vs 0.93 ; $p<0.001)$ for the detection of response to treatment in active PMR. Overall, fibrinogen showed superior correlation coefficients with the various PRO than either of the standard biomarkers ESR or CRP. In addition, standardized response means for fibrinogen, ESR, and CRP were 1.63, 1.2, and 1.05, respectively, indicating that plasma fibrinogen was the most responsive biomarker for assessment of change in disease activity.

Conclusion. VASDA and VASQOL are the most responsive PRO to changes in disease activity in PMR. In addition, plasma fibrinogen demonstrated greater responsiveness to changes in disease activity and superior correlation with the various PRO measures recorded than did the standard biomarkers ESR and CRP. (First Release April 1 2014; J Rheumatol 2014;41:931-7; doi:10.3899/jrheum.131055)

Key Indexing Terms:

POLYMYALGIA RHEUMATICA BIOMARKERS PATIENT OUTCOME MEASURES

Polymyalgia rheumatica (PMR) is a common inflammatory condition of older people ${ }^{1,2,3,4,5}$. Corticosteroids are the mainstay of treatment for PMR, although the optimal initial dose and tapering regimen are matters of ongoing debate ${ }^{6,7}$. Evidence suggests that there are 2 subsets of patients with

From the Department of Rheumatology, Mater Misericordiae University Hospital, Dublin; Department of Rheumatology, St. Vincents University Hospital, Dublin; and Department of Molecular and Cellular Therapeutics, Royal College of Surgeons, Dublin, Ireland.

E.M. McCarthy, MD; P.A. MacMullan, MD; S. Al-Mudhaffer, MD; A. Madigan, RN; S. Donnelly, MD; C.J. McCarthy, MD, Department of Rheumatology, Mater Misericordiae University Hospital; E.S. Molloy, MD, Department of Rheumatology, St. Vincents University Hospital; D. Kenny, MD, Department of Molecular and Cellular Therapeutics, Royal College of Surgeons; G.M. McCarthy, MD, Department of Rheumatology, Mater Misericordiae University Hospital.

Address correspondence to Prof. G.M. McCarthy, Rheumatology

Department, Mater Misericordiae University Hospital, Eccles Street,

Dublin 7, Ireland.E-mail: gmccarthy@rcsi.ie

Accepted for publication December 19, 2013.
PMR: those with a mild, self-limiting disease requiring 1-2 years of treatment, and others with a more chronic relapsing disease course that may require steroid treatment for several years or indefinitely ${ }^{8,9}$. Relapses are frequent in both isolated PMR and PMR associated with giant cell arteritis (GCA $)^{10,11}$. Therefore, accurate identification of disease activity in PMR is critical to balance the benefits versus serious adverse outcomes of steroid therapy ${ }^{3}$. Of all patients with PMR, 65\% have at least 1 serious corticosteroid-related event ${ }^{3}$.

Traditionally, evaluation of disease activity and response to treatment is clinical and depends on the physician's assessment in conjunction with the nonspecific inflammatory markers erythrocyte sedimentation rate (ESR) and C-reactive protein (CRP). However, the proximal pain and stiffness typical of PMR can occur in many other rheumatological illnesses in older people ${ }^{12,13}$. The traditional inflam-

Personal non-commercial use only. The Journal of Rheumatology Copyright @ 2014 . All rights reserved. 
matory markers used to assess disease activity in PMR, ESR and CRP may be within normal limits in up to $20 \%$ of patients with PMR at the time of diagnosis ${ }^{12}$. Further, normal ESR or CRP values have been observed in up to $27 \%$ and $14 \%$ of relapses, respectively, despite the increase in these variables at the time of diagnosis ${ }^{14,15,16,17}$. In addition, in a Delphi survey of relapse and remission in PMR, physician's assessment did not achieve the required agreement level highlighting its perceived poor reliability for assessment of disease activity ${ }^{18}$.

For these reasons there has been much focus on the development of validated response criteria in PMR over the past several years. A PMR activity score has been proposed and assessed in 2 patient cohorts ${ }^{19}$. However, the assessments did not include validated instruments for assessing function and quality of life (QOL). A joint European League Against Rheumatism (EULAR)/American College of Rheumatology (ACR) workforce was established, leading to the development of both classification and response criteria for $\mathrm{PMR}^{20}$.

In many rheumatic diseases, functional status and disability are important outcome measures. The ACR response criteria in RA, which combine physician-reported and patient-reported measures, have been successfully used in randomized controlled trials to facilitate approval of novel therapies ${ }^{21}$. These trials highlighted the importance of patient-reported measures of physical function and health-related $\mathrm{QOL}^{22}$ and demonstrated that patient-reported outcome (PRO) measures are less susceptible to placebo effects than physician-reported measures. PMR research studies have traditionally focused on clinical outcomes or changes in steroid treatment, whereas few studies have examined QOL despite reports of a dramatic response to steroid treatment ${ }^{23}$. There are no agreed patient-reported measures. In a study by Matteson, et al, PRO measures performed well in assessing disease activity in patients with $\mathrm{PMR}^{24}$. However, the PRO assessed in the Matteson study included morning stiffness, the value of which the international PMR/GCA study group have questioned because patients and clinicians may not reliably distinguish between pain and stiffness $^{25}$. Another major concern is that pain-related items may lack specificity given the high prevalence of osteoarthritis and degenerative pain in older people ${ }^{26}$. In addition, previous studies on patients with PMR have not demonstrated a consistent relationship between the inflammatory markers ESR and CRP, and physical QOL ${ }^{10,27}$. Recently, we have demonstrated that plasma fibrinogen is an accurate marker of disease activity in patients with active $\mathrm{PMR}^{28}$.

The goal of our current study was to prospectively investigate PRO measures in patients with PMR. We aimed to assess the responsiveness of various PRO measures to changes in disease activity and their correlation with the traditional laboratory measures of disease activity ESR and $\mathrm{CRP}$, as well as plasma fibrinogen.

\section{MATERIALS AND METHODS}

Study criteria. Ethical approval was received from the Mater Misericordiae University Hospital research ethics committee. All participants provided informed written consent. Patients were screened and prospectively recruited from the rheumatology clinic in the Mater Misericordiae University Hospital, Dublin. Patients with divided into 2 groups: active PMR (group 1) and stable/inactive PMR (group 2). Active PMR was defined as a patient with a new diagnosis of PMR with typical features or a patient with a prior history of PMR presenting with typical PMR symptoms and requiring $15 \mathrm{mg}$ of steroid to control their disease. Typical features of PMR were identified as per the Jones and Hazleman criteria ${ }^{29}$ These clinical features primarily included muscular shoulder and/or pelvic girdle pain in the absence of true muscle weakness, morning stiffness $>30$ min, symptom duration of more than 2 months unless treated, with ESR > $30 \mathrm{~mm} / \mathrm{h}$ or CRP level $>6 \mathrm{mg} / \mathrm{l}$ and a prompt and dramatic response to systemic corticosteroid therapy. Stable and inactive PMR was defined as the absence of typical PMR symptoms while taking $<5 \mathrm{mg}$ of steroid and requiring no steroid treatment for 6 weeks prior to their clinic review. Patients with concurrent PMR/GCA (biopsy proven) were included. Patients were excluded if they had either a positive rheumatoid factor and/or anticyclic citrullinated peptide, a concomitant diagnosis of another connective tissue disease, systemic infection, abnormal levels of serum creatine kinase or thyroid-stimulating hormone, or suspected underlying malignancy. Those with newly diagnosed PMR were started on $15 \mathrm{mg}$ prednisolone (GCA starting dose was $60 \mathrm{mg}$ ) and maintained on this dose for 6 weeks until their followup visit. All patients enrolled in the study were reviewed at baseline and at 6 weeks.

Clinical and laboratory analysis. The PMR disease activity score (PMR-AS) was used as the gold standard measure of disease activity at each visit for the purposes of analysis. The PMR-AS, as indicated by Leeb and $\operatorname{Bird}^{19},=\mathrm{CRP}(\mathrm{mg} / \mathrm{dl})+$ visual analog scale $(\mathrm{VAS})$ pain $(0-10$ scale $)+$ VAS physician $(0-10$ scale $)+[$ morning stiffness $(\min ) \times 0.1]+$ ability to elevate the upper limbs (EUL; 0-3 scale).

The 4 levels of semiquantitative EUL scale are as follows: $3=$ no upper limb elevation; 2 = elevation $\left(<90^{\circ}\right)$ below the shoulder girdle; $1=$ elevation $\left(90^{\circ}\right)$ up to the shoulder girdle; and $0=$ elevation $\left(>90^{\circ}\right)$ above the shoulder girdle. A PMR-AS value $<7$ indicates low disease activity, a level $<1.5$ identifying disease remission, values between 7 and 17 indicate moderate disease activity, and values $>17$ indicate high disease activity.

Standardized assays for all biomarkers were recorded at each visit for both groups. ESR was determined using the Westergren method. As ESR has been demonstrated to increase with age, particularly in females, and because most of our patients were women over the age of 50 years, the upper limit of normal considered for ESR in our study was $30 \mathrm{~mm} / \mathrm{h}$. CRP was measured using nephelometry with the upper limit of normal being $5 \mathrm{mg} / \mathrm{l}$. Plasma fibrinogen levels were evaluated at diagnosis and at the 6-week visit by a modified Clauss assay, with normal values being $<4 \mathrm{~g} / 1^{30}$.

Assessment of novel PRO measures and physical function. PRO measures including VAS pain and morning stiffness were recorded as part of the PMR-AS. In addition, a 100-mm VAS was used for recording patient assessment of disease activity (VASDA) and patient assessment of quality of life (VASQOL), with 0 indicating no disease activity or normal QOL and 100 indicating highest disease activity or worst QOL. Specifically, patients were asked to mark a vertical line on the scale in response to the question "How would you rate your PMR disease activity today?" for VASDA and "Considering all the ways that your PMR affects you, mark a vertical line corresponding to how you feel it has affected the quality of your life during the past month" for VASQOL. Data on PMR VAS pain were derived from patients' responses to the following questions, "On a scale from no pain to worst pain, how much pain are you in from your PMR today?" Functional status was assessed using the modified Health Assessment Questionnaire (mHAQ).

Statistical analysis. Normality testing was conducted using D'Agostino-Pearson test. Results for quantitative variables are reported as

Personal non-commercial use only. The Journal of Rheumatology Copyright $\odot$ 2014. All rights reserved. 
the mean \pm SD. Fisher's exact test was used in the analysis of demographic and categorical data. Between-group disease activity data were assessed using Wilcoxon signed-rank test. Standardized response means and effect size statistics, measures of a test's responsiveness to change with a higher score indicating greater responsiveness, were calculated for all 3 biomarkers ${ }^{31,32}$. Spearman's rank correlation coefficient (Spearman's rho) was used to directly compare the PMR-AS to the mHAQ, VASDA, and VASQOL, as well as to compare all biomarkers with the various PRO measured. Receiver-operator curves (ROC; a plot of percentage of true-positive results vs percentage of false-positive results, using the PMR-AS as a gold standard), predictive values, and likelihood ratios were calculated for all PRO measured. Sensitivities and specificities were calculated at different cutoff values. Statistical significance was evaluated at $\mathrm{p}<$ $0.05,2$-tailed. Statistical tests were performed using the software program Graphpad Prism 5.0.

\section{RESULTS}

Baseline demographics. Sixty patients were prospectively recruited to the study from April 2009 to June 2010. Twenty-five patients had active PMR (group 1). Thirty-five patients had inactive PMR (group 2). Only 1 patient with concomitant, biopsy-proven GCA was included; the remaining patients had isolated PMR alone. The basic demographic characteristics of the patients are shown in Table 1. There was no significant difference between the groups with regards to sex and age. There were significant differences in steroid doses between the 2 groups $(\mathrm{p}<$ $0.001)$.

Disease activity scores - comparison between groups with active and inactive disease. The mean values for the PMR-AS, PRO measures, and biomarkers are presented in Table 2. As reported, disease activity as reflected by the PMR-AS and biomarkers was significantly higher in the group with active disease (group 1) at Week 1 compared to Week 6, reflecting response to steroid therapy $(\mathrm{p}<0.001)^{28}$. Additionally, PRO measures were similarly elevated in this group at Week 1 and significantly reduced by Week 6 . Further, disease activity scores, biomarkers, and VAS measures were significantly higher at Week 1 in those with active disease compared to the inactive group (group 2) at Week 1 or $6(\mathrm{p}<0.001)$. There were no differences in the scores in group 2 between weeks 1 and 6 for any of the recorded measures. There was no significant difference between the mean PMR-AS in group 1 at Week 6 and group 2 at weeks 1 or 6 (Table 2). This indicated that, in those patients with initial active disease, disease activity was comparable to that of patients with inactive disease by Week 6 of treatment. The various PRO measures similarly identified this improvement in function in those with initial active disease.

Responsiveness of biomarkers, PMR-AS, and VAS scores to change in disease activity. Standardized response means (SRM) and effect size statistics (ESS), measures of a test's responsiveness to change (with a higher score signifying that the test is more responsive to changes in a patient's condition $^{31,32}$ ), were calculated for the various PRO measures, as well as the PMR-AS, VAS pain, and morning stiffness. VASDA and VASQOL had the highest scores, indicating they are more responsive to changes in disease activity than the previously recommended PRO measures VAS pain and morning stiffness ${ }^{24}$ as well as the PMR-AS and mHAQ (Table 3). When individual components of the mHAQ were analyzed, tasks related to rising, dressing, and grooming demonstrated the greatest responsiveness to changes in condition (SRM 1.6, 1.5, and 1.4).

When a similar analysis was performed for the individual biomarkers, both the SRM and ESS were greatest for fibrinogen, indicating that plasma fibrinogen was the most responsive biomarker for assessment of change in disease activity (Table 3 ).

Correlation of patient outcome measures with biomarkers and PMR-AS. Analysis of PMR-AS versus the PRO was performed. A strong correlation was demonstrated between the PMR-AS and all PRO. This indicates that, although the PMR-AS does not include assessments of function and QOL, it does reflect these. The strongest correlation was with VASDA. The correlation coefficients for PMR-AS versus VASDA, VASQOL, and HAQ were 0.87 ( $\mathrm{p}<0.001)$, $0.80(\mathrm{p}<0.001)$, and $0.68(\mathrm{p}<0.001)$, respectively.

Next the Spearman correlation coefficient was calculated for all the biomarkers and the PRO. All biomarkers assayed showed significant correlation with the PRO $(\mathrm{p}<0.005$; Table 4). Overall, fibrinogen showed superior correlation coefficients with the various PRO than either of the standard biomarkers (ESR or CRP), in addition to being more responsive to changes in disease activity.

Diagnostic effect of the PRO measures. ROC analysis (using the PMR-AS as a gold standard) revealed VASDA to be more specific than either HAQ $(0.95$ vs $0.85, \mathrm{p}<0.001)$ or

Table 1. Demographic characteristics of patients with polymyalgia rheumatica.

\begin{tabular}{lccc}
\hline Characteristics & Active Group & Inactive Group & $\mathrm{p}$ \\
\hline Sex & & & 0.75 \\
$\quad$ Female & 21 & 28 & \\
$\quad$ Male & 4 & 7 & 0.47 \\
Age, yrs & 70.9 & 72.7 & \\
$\quad$ SD (range) & $9.9(54-89)$ & $7.9(55-85)$ & $<0.001$ \\
Steroid dose, mg, median (range) & $15(10-60)$ & $5(0-15)$ & \\
& & & \\
\hline
\end{tabular}

Personal non-commercial use only. The Journal of Rheumatology Copyright ()$^{2014}$. All rights reserved. 
Table 2. Biomarkers and patient-reported outcome in active and inactive groups.

\begin{tabular}{|c|c|c|c|c|}
\hline \multirow[b]{2}{*}{ Biomarkers at Baseline and 6 Weeks } & \multicolumn{2}{|c|}{ Week 1} & \multicolumn{2}{|c|}{ Week 6} \\
\hline & Mean & SD (range) & Mean & SD (range) \\
\hline \multicolumn{5}{|l|}{ Active PMR group } \\
\hline $\mathrm{ESR}, \mathrm{mm} / \mathrm{h}$ & 59.6 & $30.5(10-109)$ & 24.3 & $20.1(4-79)^{+}$ \\
\hline $\mathrm{CRP}, \mathrm{mg} / \mathrm{l}$ & 45.9 & $29.1(16.5-116)$ & 12.6 & $10.5(3-38.9)^{+}$ \\
\hline Fibrinogen, g/l & 5.2 & $1.06(3.7-8.6)$ & 3.49 & $0.8(2.29-6.08)^{+}$ \\
\hline HAQ & 1.51 & $0.56(1.27-1.75)$ & 0.51 & $0.55(0.26-0.77)^{+}$ \\
\hline \multicolumn{5}{|l|}{ Inactive PMR group } \\
\hline PMR-AS & 5.2 & $5.1(0.3-18.1)$ & 5.4 & $5.2(0.4-16.6)$ \\
\hline $\mathrm{ESR}, \mathrm{mm} / \mathrm{h}$ & 17.6 & $12.2(2-63)$ & 18.3 & $11.7(2-50)$ \\
\hline CRP, mg/l & 5.2 & $3.16(3-16.1)$ & 6 & $4.79(3-20.1)$ \\
\hline Fibrinogen, g/l & 3.23 & $0.59(1.64-4.19)$ & 3.24 & $0.53(2.15-4.24)$ \\
\hline
\end{tabular}

${ }^{+} \mathrm{p}=0.001$ compared with baseline values. PMR: polymyalgia rheumatica; AS: activity score; ESR: erythrocyte sedimentation rate; CRP: C-reactive protein; VAS: visual analog scale; DA: disease activity; QOL: quality of life; HAQ: Health Assessment Questionnaire.

Table 3. Responsiveness of assessed measures and biomarkers to changes in disease activity.

\begin{tabular}{lcc}
\hline $\begin{array}{l}\text { Patient-reported } \\
\text { Outcome Measures }\end{array}$ & $\begin{array}{c}\text { Standardized } \\
\text { Response Means }\end{array}$ & Effect Size \\
\hline VASDA & 1.76 & 2.52 \\
VASQOL & 1.74 & 2.27 \\
PMR-AS & 1.54 & 1.84 \\
VAS pain & 1.52 & 1.7 \\
HAQ & 1.36 & 1.65 \\
Morning stiffness & 0.89 & 0.96 \\
Biomarkers & & \\
$\quad$ Fibrinogen & 1.63 & 1.6 \\
$\quad$ ESR & 1.2 & 1.15 \\
CRP & 1.05 & 1.14 \\
\hline
\end{tabular}

PMR: polymyalgia rheumatica; AS: activity score; VAS: visual analog scale; DA: disease activity; QOL: quality of life; HAQ: Health Assessment Questionnaire; ESR: erythrocyte sedimentation rate; CRP: C-reactive protein.

Table 4. Correlation coefficients of the biomarkers and patient-reported outcome measures.

\begin{tabular}{lccc}
\hline Biomarkers & HAQ & VASDA & VASQOL \\
\hline Fibrinogen & 0.51 & 0.64 & 0.61 \\
ESR & 0.45 & 0.57 & 0.57 \\
CRP & 0.39 & 0.62 & 0.64 \\
\hline
\end{tabular}

VAS: visual analog scale; DA: disease activity; QOL: quality of life; HAQ: Health Assessment Questionnaire; ESR: erythrocyte sedimentation rate; CRP: C-reactive protein.

VASQOL $(0.95$ vs $0.93, p<0.001)$ for the detection of response to treatment in patients with active PMR (Figure 1).

Because HAQ is measured on a $0-3$ scale and VAS on a
0-10 scale, we assumed a sensitivity of $92 \%$ for all 3 PRO. Assuming the same sensitivity levels revealed that in addition, VASDA had a superior likelihood ratio for detecting active PMR. VASDA likelihood ratio was 10.43 vs 3.68 and 3.33 for VASQOL and HAQ, respectively ( $<<$ $0.001)$.

\section{DISCUSSION}

The results of our investigation demonstrate for the first time the usefulness of PRO measures, VASDA, and VASQOL in assessing disease activity in patients with PMR. In particular, VASDA and VASQOL were more responsive to changes in disease activity than the traditionally accepted PRO measures of morning stiffness and VAS pain. In addition, both tools, as well as the HAQ, demonstrated strong correlation with the PMR-AS, confirming that although the PMR-AS does not assess patient function/perception of disease activity, it does reflect them. Thus VASDA and VASQOL may help rheumatologists and general practitioners to better monitor PMR activity and to manage glucocorticoid tapering, particularly because these tests are easily administered in clinics. Previous work conducted by our group identified plasma fibrinogen as an accurate marker of disease activity in $\mathrm{PMR}^{28}$. Additionally, our current study demonstrates that plasma fibrinogen is more responsive to changes in disease activity and shows better correlation with the various PRO measures recorded than the standard biomarkers ESR and CRP, further highlighting its potential utility in assessing disease activity in PMR.

To safely guide glucocorticoid taper and detect flares in patients with known PMR, physicians rely on their assessment of a patient's symptoms and serial measure- 


\section{ROC of PROs in PMR: wk1 v wk6}

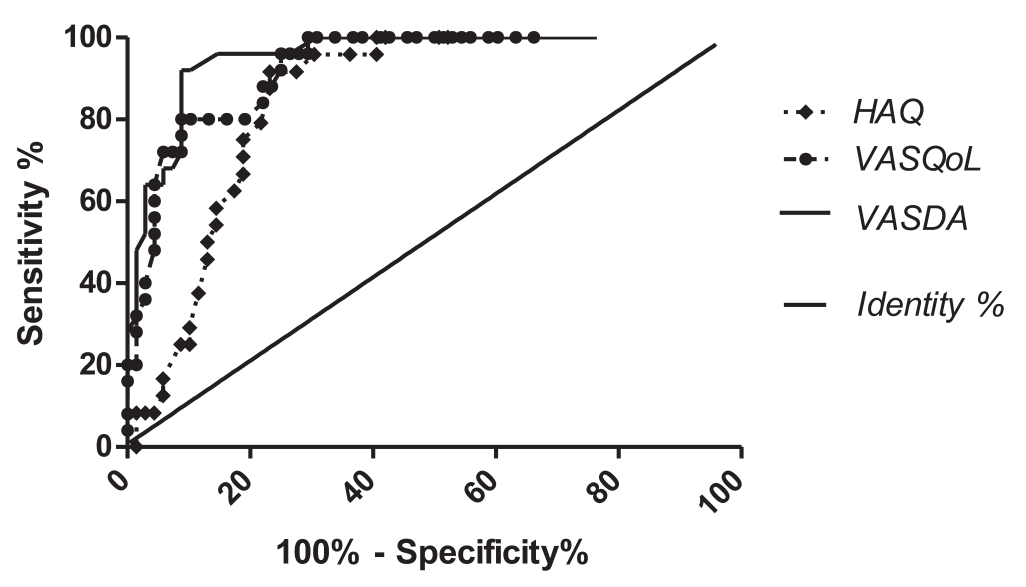

Figure 1. Receiver-operator curves (ROC) for visual analog scale disease activity (VASDA), quality of life (VASQOL), and Health Assessment Questionnaire (HAQ). PRO: patient-reported outcome; PMR: polymyalgia rheumatica.

ments of the nonspecific inflammatory markers ESR and CRP. However, physician assessment of response to treatment in those with active PMR is unreliable, and therefore has been excluded for the agreed ACR/EULAR response criteria for disease activity in $\mathrm{PMR}^{18}$. In addition, ESR and CRP can be normal at the time of flare ${ }^{14,15,16,17}$. For this reason there is increasing interest in PRO measures in PMR. PRO measures are accepted as valid scientific outcome measures of musculoskeletal conditions regardless of laboratory findings and are included in trials of biologic agents in inflammatory arthritis. As new therapies become available for PMR, trials of these agents will need to include the patients' perspective on the efficacy of treatment, thus the need for reliable, validated, and easily administered tools.

In this regard, our study suggests that measurement of VASDA and VASQOL may assist both patients and physicians in assessing disease activity. The majority of patients with PMR are managed solely in a primary care setting. Therefore, any tool that can be easily administered and is more responsive to changes in disease activity than traditional patient measures of disease activity, VAS pain, and morning stiffness, would be helpful. VASDA in particular was highly specific and exhibited a high likelihood ratio for detecting response to treatment, as well as demonstrating a strong correlation with the gold standard PMR-AS. Traditional patient outcome measures assessed in PMR have focused on pain and stiffness ${ }^{24,27}$. Elderly patients with multiple musculoskeletal problems may have difficulty distinguishing between PMR-related disability and more fixed disabilities such as osteoarthritis. These concerns, previously highlighted by the PMR working group and supported by our findings that pain and stiffness are among the least responsive measures of change in disease activity $^{25}$, may be addressed by using PRO measures such as VASDA and VASQOL, which exclude pain or morning stiffness. Although HAQ was the least responsive tool assessed in our study, detailed analysis of its component parts revealed that rising from a seated position was the most responsive individual measure of change rather than the expected measures of upper limb/shoulder function, such as dressing and grooming. This highlights bilateral hip involvement as a potential new indicator of response to treatment in PMR, a finding that warrants further investigation.

In addition to patient assessment measures, the joint EULAR/ACR study has mandated measurement of a minimum of 1 inflammatory marker to help guide decisions regarding disease activity ${ }^{24}$. However, none of the currently available biomarkers are specific for disease. In support of this, a small number of patients in the inactive arm of our study presented with elevated inflammatory markers despite the complete absence of PMR symptoms and no exacerbation of their symptoms on followup. Similarly, previous work done on other potential novel biomarkers, such as von Willebrand factor, demonstrated that although levels of von Willebrand factor are elevated in active disease they do not normalize rapidly with the initiation of steroid therapy, suggesting that measurement of these components is unlikely to be useful in the day-to-day management of disease, once more highlighting the challenge of identifying robust biomarkers for $\mathrm{PMR}^{33,34}$. Therefore, any biomarker that is superior to the current standard markers would be useful if it helped guide more accurate therapeutic decisions with regards to disease remission and relapse. Our study highlights the superior responsiveness of plasma fibrinogen compared to the traditional biomarkers ESR and CRP for assessing changes in disease activity. In PMR, interleukin 6 (IL-6) levels closely correlate with the systemic manifestations of the disease ${ }^{35}$.

$$
\text { Personal non-commercial use only. The Journal of Rheumatology Copyright @ } 2014 \text {. All rights reserved. }
$$


Theoretically, IL-6 provides a more accurate measure of tissue pathologic processes because it is one of the proinflammatory cytokines released in vascular lesions ${ }^{36}$. Plasma fibrinogen has also been shown to be exquisitely related to IL-6 production ${ }^{37}$. Thus, fibrinogen may be thought of as a downstream reflection of the pathological events that are driven by IL- 6 production ${ }^{36}$. Measurement of plasma fibrinogen may, therefore, enhance accuracy of diagnosis and therapeutic decisions, in particular because it also better reflects PRO measures than do ESR and CRP.

Our study has a number of limitations. As a pilot study, the number of patients enrolled was small. Thus, validation of our results is required using a larger cohort of patients followed for a longer time period, in particular to examine the role of fibrinogen in those patients presenting with normal ESR and CRP at the time of relapse. Additionally, inclusion of ultrasound evaluation of patients at the time of PRO and fibrinogen assessment may have allowed for enhanced understanding of how these tools reflect specific joint/bursa involvement and patient function. Finally, as with any clinical observation, further detailed laboratory analysis is required to elucidate the mechanisms by which plasma fibrinogen appears to be more responsive to changes in disease activity than CRP, in particular because the production of both biomarkers is driven by IL-6.

Nonetheless, our study supports the relevance of PRO measures in assessing PMR activity in everyday practice. Standardized patient-reported measures would be welcomed if they led to more accurate assessment of disease activity and appropriate adjustment of steroid dose. In this regard, VASDA seems the most promising tool for assessing disease activity in PMR, particularly because it is easily administered in the clinical setting and is not currently part of assessment criteria. Further, our data suggest that measurement of fibrinogen as an adjunct to ESR and CRP in patients with suspected active PMR will enhance accuracy of diagnosis and guide therapeutic decisions, especially because it better reflects PRO in PMR than do the standard biomarkers ESR or CRP. Inclusion of both plasma fibrinogen and VASDA in future studies of novel markers of disease activity and responsiveness in PMR is warranted.

\section{REFERENCES}

1. Lawrence RC, Felson DT, Helmick CG, Arnold LM, Choi H, Deyo RA, et al. Estimates of the prevalence of arthritis and other rheumatic conditions in the United States. Part II. Arthritis Rheum 2008;58:26-35

2. Walsh LJ, Wong CA, Pringle M, Tattersfield AE. Use of oral corticosteroids in the community and the prevention of secondary osteoporosis: a cross sectional study. BMJ 1996;313:344-6.

3. Gabriel SE, Sunku J, Salvarani C, O'Fallon WM, Hunder GG. Adverse outcomes of antiinflammatory therapy among patients with polymyalgia rheumatica. Arthritis Rheum 1997;40:1873-8.

4. Dasgupta B, Matteson EL, Maradit-Kremers H. Management guidelines and outcome measures in polymyalgia rheumatica (PMR). Clin Exp Rheumatol 2007;6 Suppl 47:130-6.
5. Dasgupta B, Borg FA, Hassan N, Barraclough K, Bourke B, Fulcher J, et al. BSR and BHPR guidelines for the management of polymyalgia rheumatica. Rheumatology 2010;49:186-90.

6. Kremers HM, Reinalda MS, Crowson CS, Zinsmeister AR, Hunder GG, Gabriel SE. Use of physician services in a population-based cohort of patients with polymyalgia rheumatica over the course of their disease. Arthritis Rheum 2005;53:395-403.

7. Gonzalez-Gay MA, Garcia-Porrua C, Vazquez-Caruncho M, Dababneh A, Hajeer A, Ollier WE. The spectrum of polymyalgia rheumatica in northwestern Spain: incidence and analysis of variables associated with relapse in a 10 year study. J Rheumatol 1999;26:1326-32.

8. Ayoub WT, Franklin CM, Torretti D. Polymyalgia rheumatica. Duration of therapy and long-term outcome. Am J Med 1985;79:309-15.

9. Narváez J, Nolla-Solé JM, Clavaguera MT, Valverde-García J, Roig-Escofet D. Longterm therapy in polymyalgia rheumatica: effect of coexistent temporal arteritis. J Rheumatol 1999; 26:1945-52.

10. Salvarani C, Cantini F, Niccoli L, Macchioni P, Consonni D, Bajocchi G, et al. Acute-phase reactants and the risk of relapse/recurrence in polymyalgia rheumatica: a prospective followup study. Arthritis Rheum 2005;53:33-8.

11. Kremers HM, Reinalda MS, Crowson CS, Zinsmeister AR, Hunder GG, Gabriel SE. Relapse in a population based cohort of patients with polymyalgia rheumatica. J Rheumatol 2005;32:65-73.

12. Salvarani C, Cantini F, Boiardi L, Hunder GG. Polymyalgia rheumatica and giant-cell arteritis. N Engl J Med 2002;347:261-71.

13. Weyand CM, Goronzy JJ. Giant-cell arteritis and polymyalgia rheumatica. Ann Intern Med 2003;139:505-15.

14. Caporali R, Cimmino MA, Ferraccioli G, Gerli R, Klersy C, Salvarani C, et al. Prednisone plus methotrexate for polymyalgia rheumatica: a randomized, double-blind, placebo-controlled trial. Ann Intern Med 2004;141:493-500.

15. Cantini F, Salvarani C, Olivieri I, Macchioni L, Ranzi A, Niccoli L, et al. Erythrocyte sedimentation rate and $\mathrm{C}$-reactive protein in the evaluation of disease activity and severity in polymyalgia rheumatica: a prospective follow-up study. Semin Arthritis Rheum 2000;30:17-24.

16. Bengtsson BA, Malmvall BE. Prognosis of giant cell arteritis including temporal arteritis and polymyalgia rheumatica. A follow-up study on ninety patients treated with corticosteroids. Acta Med Scand 1981;209:337-45.

17. von Knorring J. Treatment and prognosis in polymyalgia rheumatica and temporal arteritis. A ten-year survey of 53 patients. Acta Med Scand 1979;205:429-35.

18. Dejaco C, Duftner C, Cimmino MA, Dasgupta B, Salvarani C, Crowson CS, et al. Definition of remission and relapse in polymyalgia rheumatica: data from a literature search compared with a Delphi-based expert consensus. Ann Rheum Dis 2011;70:447-53.

19. Leeb BF, Bird HA. A disease activity score for polymyalgia rheumatica. Ann Rheum Dis 2004;63:1279-83.

20. Dasgupta B, Cimmino MA, Maradit-Kremers H, Schmidt WA, Schirmer M, Salvarani C, et al. 2012 provisional classification criteria for polymyalgia rheumatica: a European League Against Rheumatism/American College of Rheumatology collaborative initiative. Ann Rheum Dis 2012;71:484-92.

21. Felson DT, Anderson JJ, Boers M, Bombardier C, Chernoff M, Fried B, et al. The American College of Rheumatology preliminary core set of disease activity measures for rheumatoid arthritis clinical trials. The Committee on Outcome Measures in Rheumatoid Arthritis Clinical Trials. Arthritis Rheum 1993; 36:729-40.

22. Buchbinder R, Bombardier C, Yeung M, Tugwell P. Which outcome

Personal non-commercial use only. The Journal of Rheumatology Copyright (c) 2014. All rights reserved. 
measures should be used in rheumatoid arthritis clinical trials? Clinical and quality-of-life measures' responsiveness to treatment in a randomized controlled trial. Arthritis Rheum 1995;38:1568-80.

23. Kalke S, Mukerjee D, Dasgupta B. A study of the health assessment questionnaire to evaluate functional status in polymyalgia rheumatica. Rheumatology 2000;39:883-5.

24. Matteson EL, Maradit-Kremers H, Cimmino MA, Schmidt WA, Schirmer M, Salvarani C, et al. Patient-reported outcomes in polymyalgia rheumatica. J Rheumatol 2012;39:795-803.

25. Dasgupta B, Salvarani C, Schirmer M, Crowson CS, Maradit-Kremers H, Hutchings A, et al. Developing classification criteria for polymyalgia rheumatica: comparison of views from an expert panel and wider survey. J Rheumatol 2008;35:270-7.

26. Zhang Y, Jordan JM. Epidemiology of osteoarthritis. Rheum Dis Clin North Am 2008;34:515-29.

27. Hutchings A, Hollywood J, Lamping DL, Pease CT, Chakravarty K, Silverman B, et al. Clinical outcomes, quality of life, and diagnostic uncertainty in the first year of polymyalgia rheumatica. Arthritis Rheum 2007;57:803-9.

28. McCarthy EM, Macmullan PA, Al-Mudhaffer S, Madigan A, Donnelly S, McCarthy CJ, et al. Plasma fibrinogen is an accurate marker of disease activity in patients with polymyalgia rheumatica. Rheumatology 2013;52:465-71.

29. Jones JG, Hazleman BL. Prognosis and management of polymyalgia rheumatica. Ann Rheum Dis 1981;40:1-5.
30. Mackie IJ, Kitchen S, Machin SJ, Lowe GD. Guidelines on fibrinogen assays. Br J Haematol 2003;121:396-404.

31. Guyatt G, Walter S, Norman G. Measuring change over time: assessing the usefulness of evaluative instruments. J Chronic Dis 1987;40:171-8.

32. Husted JA, Gladman DD, Cook RJ, Farewell VT. Responsiveness of health status instruments to changes in articular status and perceived health in patients with psoriatic arthritis. J Rheumatol 1998;25:2146-55.

33. Persellin ST, Daniels TM, Rings LJ, Kazmier FJ, Bowie EJ, Hunder GG. Factor VIII-von Willebrand factor in giant cell arteritis and polymyalgia rheumatica. Mayo Clin Proc 1985;60:457-62.

34. Uddhammar A, Rantapåå-Dahlqvist S, Nilsson TK. Plasminogen activator inhibitor and von Willebrand factor in polymyalgia rheumatica. Clin Rheumatol 1992;11:211-5.

35. Alvarez-Rodriguez L, Lopez-Hoyos M, Mata C, Marin MJ, Calvo-Alen J, Blanco R, et al. Circulating cytokines in active polymyalgia rheumatica. Ann Rheum Dis 2010;69:263-9.

36. Weyand CM, Fulbright JW, Hunder GG, Evans JM, Goronzy JJ. Treatment of giant cell arteritis: interleukin-6 as a biologic marker of disease activity. Arthritis Rheum 2000;43:1041-8.

37. Heinrich PC, Castell JV, Andus T. Interleukin-6 and the acute phase response. Biochem J 1990;265:621-36. 